\title{
Periodic solution for $p$-Laplacian Rayleigh equation with attractive singularity and time-dependent deviating argument
}

\section{Zhibo Cheng ${ }^{1,2^{*}}$, Zhonghua $\mathrm{Bi}^{1}$ and Shaowen Yao ${ }^{{ }^{*}}$}

\section{"Correspondence:}

czbo@hpu.edu.cn;

yaoshaowen@hpu.edu.cn

${ }^{1}$ School of Mathematics and

Information Science, Henan

Polytechnic University, Jiaozuo,

China

${ }^{2}$ Department of Mathematics,

Sichuan University, Chengdu, China

\begin{abstract}
In this paper, we consider a p-Laplacian singular Rayleigh equation with time-dependent deviating argument

$$
\left(\varphi_{p}\left(x^{\prime}(t)\right)\right)^{\prime}+f\left(t, x^{\prime}(t)\right)+g(t, x(t-\sigma(t)))=e(t),
$$

where $g$ has an attractive singularity at $x=0$. Using the Manásevich-Mawhin continuation theorem, we prove that the equation has at least one $T$-periodic solution.
\end{abstract}

MSC: $34 \mathrm{~K} 13 ; 34 \mathrm{C} 25$

Keywords: Rayleigh equation; Periodic solution; Attractive singularity; $p$-Laplacian; Time-dependent deviating argument

\section{Introduction}

In the past years, researchers paid much attention to investigating the problem of periodic solutions for second-order equations with singularities (see [1-16]). Among those studies, the study of properties of repulsive singularities can be traced back to 1996. Zhang [1] discussed the existence of positive periodic solutions of the following Liénard equation with singularity:

$$
x^{\prime \prime}(t)+f(x(t)) x^{\prime}(t)+g(t, x(t))=0
$$

where $g(t, x(t))$ may be unbounded as $x \rightarrow 0^{+}$. Equation (1.1) is of repulsive type (resp. attractive type) if $g(t, x(t)) \rightarrow-\infty$ (resp. $g(t, x(t)) \rightarrow+\infty)$ as $x \rightarrow 0^{+}$. Using Mawhin's continuation theorem, the author proved that Eq. (1.1) has at least one $T$-periodic solution.

Zhang's work has attracted much attention of many specialists in differential equations. In 2014, Wang [2] investigated the existence of positive periodic solutions of the following Liénard equation with singularity and deviating argument:

$$
x^{\prime \prime}(t)+f(x(t)) x^{\prime}(t)+g(t, x(t-\sigma))=0,
$$

(c) The Author(s) 2018. This article is distributed under the terms of the Creative Commons Attribution 4.0 International License (http://creativecommons.org/licenses/by/4.0/), which permits unrestricted use, distribution, and reproduction in any medium, provided you give appropriate credit to the original author(s) and the source, provide a link to the Creative Commons license, and indicate if changes were made. 
where $g$ satisfies the same conditions as in Eq. (1.1), and $\sigma$ is a constant such that $0 \leq \sigma<T$. In 2017, $\mathrm{Lu}$ [3] considered the existence of positive periodic solutions of the following Liénard equation with singularity:

$$
x^{\prime \prime}(t)+f(x(t)) x^{\prime}(t)-g(x(t))+\varphi(t) x(t)=h(t)
$$

where $g(x)$ is singular at $x=0$, and $\varphi$ and $h$ are $T$-periodic functions. The authors found a new method for estimating a lower a priori bounds of the periodic solutions to the given equation. Besides, many articles have been published about Liénard equation with repulsive singularity (see [4-13]).

Recently, some good deal of works have been performed on the existence of periodic solutions of Rayleigh equations with singularity (see [14-16]). Wang and Ma [16] in 2015 studied the Rayleigh equation with repulsive singularity

$$
x^{\prime \prime}(t)+f\left(t, x^{\prime}(t)\right)+g(x(t))=p(t)
$$

where $g$ has a repulsive singularity at the origin. The authors obtained that the given equation has at least one $2 \pi$-periodic solution.

All the aforementioned results are related to equations with repulsive singularity or equations with time-independent deviating argument. Naturally, a new question arises: how the Rayleigh equation with attractive singularity works on time-dependent deviating argument? Besides practical interests, the topic has obvious intrinsic theoretical significance. To answer this question, in this paper, applying the Manásevich-Mawhin continuation theorem, we consider the existence of positive periodic solutions for the following Rayleigh equation with attractive singularity and time-dependent deviating argument:

$$
\left(\varphi_{p}\left(x^{\prime}(t)\right)\right)^{\prime}+f\left(t, x^{\prime}(t)\right)+g(t, x(t-\sigma(t)))=e(t)
$$

where $\varphi_{p}: \mathbb{R} \rightarrow \mathbb{R}$ is given by $\varphi_{p}(s)=|s|^{p-2} s$ with constant $p>1, f \in C(\mathbb{R} \times \mathbb{R}, \mathbb{R})$, $e \in C(\mathbb{R}, \mathbb{R}), f\left(t, x^{\prime}(t)\right)$ and $e(t)$ are $T$-periodic with respect to variable $t, \int_{0}^{T} e(t) d t=0$, $g(t, x)=g_{0}(x)+g_{1}(t, x)$ with $g_{0} \in C((0, \infty) ; \mathbb{R})$ and an $L^{2}$-Carathéodory function $g_{1}, g_{0}$ has an attractive singularity at $x=0$, that is,

$$
\int_{0}^{1} g_{0}(x) d x=+\infty
$$

and $\sigma \in C^{1}(\mathbb{R}, \mathbb{R})$ is a $T$-periodic function such that $\sigma^{\prime}(t)<1$. Obviously, the attractivity condition $\lim _{x \rightarrow 0^{+}} \int_{x}^{1} g_{0}(s) d s=+\infty$ contradicts the repulsive singularity. Therefore, the methods of $[1,2,16]$ are no longer applicable to prove the existence of periodic solutions for Eq. (1.3) with attractive singularity. So we need to find a new method to get over it.

In this paper, we give a new condition for $g(t, x)$ in Eq. (1.3) with attractive singularity, namely, $-g(t, x) \leq a x^{p-1}+b$, where $a, b$ are positive constants. Therefore, by estimating $a$ priori bounds of periodic solutions and the Manásevich-Mawhin continuation theorem we prove that Eq. (1.3) has at least one $T$-periodic solution. 


\section{Periodic solution for Eq. (1.3)}

We consider the $T$-periodic boundary value problem

$$
\left(\varphi_{p}\left(x^{\prime}(t)\right)\right)^{\prime}=\tilde{f}\left(t, x(t), x^{\prime}(t)\right)
$$

where $\tilde{f}:[0, T] \times \mathbb{R} \times \mathbb{R} \rightarrow \mathbb{R}$ is assumed to be Carathéodory.

Lemma 2.1 (Manásevich-Mawhin [17]) Let $\Omega$ be an open bounded set in $C_{T}^{1}:=\{x \in$ $\left.C^{1}(\mathbb{R}, \mathbb{R}): x(t+T)-x(t) \equiv 0\right\}$. Suppose that:

(i) For each $\lambda \in(0,1)$, the problem

$$
\left(\varphi_{p}\left(x^{\prime}(t)\right)\right)^{\prime}=\lambda \tilde{f}\left(t, x(t), x^{\prime}(t)\right), \quad x(0)=x(T), \quad x^{\prime}(0)=x^{\prime}(T),
$$

has no solution on $\partial \Omega$.

(ii) The equation

$$
F(a):=\frac{1}{T} \int_{0}^{T} \tilde{f}(t, a, 0) d t=0
$$

has no solution on $\partial \Omega \cap \mathbb{R}$.

(iii) The Brouwer degree

$$
\operatorname{deg}\{F, \Omega \cap \mathbb{R}, 0\} \neq 0 .
$$

Then the periodic boundary value problem (2.1) has at least one T-periodic solution on $\bar{\Omega}$.

Next, applying the Manśevich-Mawhin continuation theorem, we prove the following theorems. Define

$$
\|x\|:=\max _{t \in[0, T]}|x(t)|, \quad\left\|x^{\prime}\right\|:=\max _{t \in[0, T]}\left|x^{\prime}(t)\right| .
$$

Theorem 2.1 Assume that the following conditions are satisfied:

$\left(\mathrm{H}_{1}\right) f(t, 0)=0$, and there exists a constant $K>0$ such that $|f(t, u)| \leq K$ for $(t, u) \in \mathbb{R} \times \mathbb{R}$.

$\left(\mathrm{H}_{2}\right)$ There exists positive constants $D_{1}$ and $D_{2}$ with $0<D_{2}<D_{1}$ such that $g(t, x)<-K$ for $(t, x) \in \mathbb{R} \times\left(D_{1},+\infty\right)$ and $g(t, x)>K$ for $(t, x) \in \mathbb{R} \times\left(0, D_{2}\right)$.

$\left(\mathrm{H}_{3}\right)$ There exist positive constants $a$ and $b$ such that

$$
-g(t, x) \leq a x^{p-1}+b \quad \text { for }(t, x) \in \mathbb{R} \times(0,+\infty) .
$$

Then Eq. (1.3) has at least one solution with period $T$ if $2 a T^{p}<1$.

Proof Consider the equation

$$
\left(\varphi_{p}\left(x^{\prime}(t)\right)\right)^{\prime}+\lambda f\left(t, x^{\prime}(t)\right)+\lambda g(t, x(t-\sigma(t)))=\lambda e(t) .
$$

Firstly, we will claim that the set of all $T$-periodic solution of Eq. (2.2) is bounded. Let $x \in C_{T}:=\{x \in C(\mathbb{R}, \mathbb{R}): x(t+T)-x(t) \equiv 0\}$ be an arbitrary $T$-periodic solution of Eq. (2.2). 
Integrating both sides of Eq. (2.2) over [0,T], we have

$$
\int_{0}^{T}\left(\varphi_{p}\left(x^{\prime}(t)\right)\right)^{\prime} d t+\lambda \int_{0}^{T} f\left(t, x^{\prime}(t)\right) d t+\lambda \int_{0}^{T} g(t, x(t-\sigma(t))) d t=\lambda \int_{0}^{T} e(t) d t .
$$

Since $\int_{0}^{T}\left(\varphi_{p}\left(x^{\prime}(t)\right)\right)^{\prime} d t=0$ and $\int_{0}^{T} e(t) d t=0$, we have

$$
\int_{0}^{T}\left(f\left(t, x^{\prime}(t)\right)+g(t, x(t-\sigma(t)))\right) d t=0
$$

From Eq. (2.3) and condition $\left(\mathrm{H}_{1}\right)$ we have

$$
-K T<\int_{0}^{T} g(t, x(t-\sigma(t))) d t<K T .
$$

Then by condition $\left(\mathrm{H}_{2}\right)$ we know that there exist two points $\xi_{1}, \eta_{1} \in[0, T]$ such that

$$
x\left(\xi_{1}\right) \leq D_{1}, x\left(\eta_{1}-\sigma\left(\eta_{1}\right)\right)>D_{2} .
$$

Since $\|x\| \leq x\left(\xi_{1}\right)+T^{\frac{1}{q}}\left(\int_{0}^{T}\left|x^{\prime}(t)\right|^{p} d t\right)^{\frac{1}{p}}$, we have

$$
\|x\| \leq D_{1}+T^{\frac{1}{q}}\left(\int_{0}^{T}\left|x^{\prime}(t)\right|^{p} d t\right)^{\frac{1}{p}} .
$$

Multiplying both sides of Eq. (2.2) by $x(t)$ and integrating over the interval $[0, T]$, we get

$$
\begin{aligned}
& \int_{0}^{T}\left(\varphi_{p}\left(x^{\prime}(t)\right)\right)^{\prime} x(t) d t+\lambda \int_{0}^{T} f\left(t, x^{\prime}(t)\right) x(t) d t+\lambda \int_{0}^{T} g(t, x(t-\sigma(t))) x(t) d t \\
& \quad=\lambda \int_{0}^{T} e(t) x(t) d t .
\end{aligned}
$$

Substituting $\int_{0}^{T}\left(\varphi_{p}\left(x^{\prime}(t)\right)\right)^{\prime} x(t) d t=-\int_{0}^{T}\left|x^{\prime}(t)\right|^{p} d t$ into Eq. (2.5), we have

$$
\begin{aligned}
-\int_{0}^{T}\left|x^{\prime}(t)\right|^{p} d t= & -\lambda \int_{0}^{T} f\left(t, x^{\prime}(t)\right) x(t) d t-\lambda \int_{0}^{T} g(t, x(t-\sigma(t))) x(t) d t \\
& +\lambda \int_{0}^{T} e(t) x(t) d t .
\end{aligned}
$$

Thus we have

$$
\begin{aligned}
\int_{0}^{T}\left|x^{\prime}(t)\right|^{p} d t \leq & \lambda \int_{0}^{T}\left|f\left(t, x^{\prime}(t)\right)\right||x(t)| d t+\lambda \int_{0}^{T}|g(t, x(t-\sigma(t)))||x(t)| d t \\
& +\lambda \int_{0}^{T}|e(t)||x(t)| d t \\
\leq & K T\|x\|+\|x\| \int_{0}^{T}|g(t, x(t-\sigma(t)))| d t+\|x\| \int_{0}^{T}|e(t)| d t .
\end{aligned}
$$


From Eq. (2.3) and condition $\left(\mathrm{H}_{3}\right)$ we have

$$
\begin{aligned}
& \int_{0}^{T}|g(t, x(t-\sigma(t)))| d t \\
& \quad=\int_{g(t, x(t-\sigma(t)))>0} g^{+}(t, x(t-\sigma(t))) d t-\int_{g(t, x(t-\sigma(t))) \leq 0} g^{-}(t, x(t-\sigma(t))) d t \\
& \quad=-2 \int_{g(t, x(t-\sigma(t))) \leq 0} g^{-}(t, x(t-\sigma(t))) d t+\int_{0}^{T} f\left(t, x^{\prime}(t)\right) d t \\
& \quad \leq 2 \int_{0}^{T}\left(a x^{p-1}(t)+b\right) d t+\int_{0}^{T}\left|f\left(t, x^{\prime}(t)\right)\right| d t \\
& \quad \leq 2 a T\|x\|^{p-1}+2 b T+K T,
\end{aligned}
$$

where $g^{-}:=\min \{g(t, x(t-\sigma(t))), 0\}$. Substituting Eq. (2.7) into Eq. (2.6), we have

$$
\int_{0}^{T}\left|x^{\prime}(t)\right|^{p} d t \leq 2 a T\|x\|^{p}+\|x\|(2 K T+2 b T+\|e\| T) .
$$

Substituting Eq. (2.4) into Eq. (2.8), we get

$$
\begin{aligned}
\int_{0}^{T}\left|x^{\prime}(t)\right|^{p} d t \leq & 2 a T\left(D_{1}+T^{\frac{1}{q}}\left(\int_{0}^{T}\left|x^{\prime}(t)\right|^{p} d t\right)^{\frac{1}{p}}\right)^{p} \\
& +(2 b T+\|e\| T+2 K T)\left(D_{1}+T^{\frac{1}{q}}\left(\int_{0}^{T}\left|x^{\prime}(t)\right|^{p} d t\right)^{\frac{1}{p}}\right) \\
\leq & 2 a T\left(T^{\frac{p}{q}} \int_{0}^{T}\left|x^{\prime}(t)\right|^{p} d t+(1+p) D_{1} T^{\frac{p-1}{q}}\left(\int_{0}^{T}\left|x^{\prime}(t)\right|^{p} d t\right)^{\frac{p-1}{p}}\right) \\
& \left.+(2 b T+\|e\| T+2 K T) D_{1}\right) \\
& +(2 b T+\|e\| T+2 K T) T^{\frac{1}{q}}\left(\int_{0}^{T}\left|x^{\prime}(t)\right|^{p} d t\right)^{\frac{1}{p}} \\
= & 2 a T^{\frac{p+q}{q}} \int_{0}^{T}\left|x^{\prime}(t)\right|^{p} d t+2 a T^{\frac{p+q-1}{q}}(1+p) D_{1}\left(\int_{0}^{T}\left|x^{\prime}(t)\right|^{p} d t\right)^{\frac{p-1}{p}} \\
& +(2 b T+\|e\| T+2 K T) D_{1} \\
& +(2 b T+\|e\| T+2 K T) T^{\frac{1}{q}}\left(\int_{0}^{T}\left|x^{\prime}(t)\right|^{p} d t\right)^{\frac{1}{p}},
\end{aligned}
$$

since $(1+x)^{p} \leq 1+(1+p) x$ for $x \in[0, \delta]$, where $\delta$ is a given positive constant depending only on $p>0$. Thus we have

$$
\begin{aligned}
& \left(D_{1}+T^{\frac{1}{q}}\left(\int_{0}^{T}\left|x^{\prime}(t)\right|^{p} d t\right)^{\frac{1}{p}}\right)^{p} \\
& \quad \leq T^{\frac{p}{q}} \int_{0}^{T}\left|x^{\prime}(t)\right|^{p} d t+(1+p) D_{1} T^{\frac{p-1}{q}}\left(\int_{0}^{T}\left|x^{\prime}(t)\right|^{p} d t\right)^{\frac{p-1}{p}} .
\end{aligned}
$$


Since $\frac{1}{p}+\frac{1}{q}=1$, we get $2 a T^{\frac{p+q}{q}}=2 a T^{p}<1$. It is easy to see that there exists a constant $M_{1}^{\prime}>0$ (independent of $\lambda$ ) such that

$$
\int_{0}^{T}\left|x^{\prime}(t)\right|^{p} d t \leq M_{1}^{\prime}
$$

From Eq. (2.4) and Eq. (2.10) we have

$$
\|x\| \leq D_{1}+T^{\frac{1}{q}}\left(\int_{0}^{T}\left|x^{\prime}(t)\right|^{p} d t\right)^{\frac{1}{p}} \leq D_{1}+T^{\frac{1}{q}}\left(M_{1}^{\prime}\right)^{\frac{1}{p}}:=M_{1} .
$$

Since $x(t)$ is $T$-periodic, there exists a point $t_{0} \in(0, T)$ such that $x^{\prime}\left(t_{0}\right)=0$, whereas $\varphi_{p}(0)=0$. Hence, from Eq. (2.7) and Eq. (2.11) we have that

$$
\begin{aligned}
\left|\varphi_{p}\left(x^{\prime}(t)\right)\right| & =\left|\int_{t_{0}}^{t}\left(\varphi_{p}\left(x^{\prime}(s)\right)\right)^{\prime} d s\right| \\
& \leq \lambda \int_{0}^{T}\left|f\left(t, x^{\prime}(t)\right)\right| d t+\lambda \int_{0}^{T}|g(t, x(t-\sigma(t)))| d t+\lambda \int_{0}^{T}|e(t)| d t \\
& \leq 2 K T+2 a T M_{1}^{p-1}+2 b T+T\|e\|:=M_{2}^{\prime} .
\end{aligned}
$$

Next, we claim that there exists a positive constant $M_{2}>M_{2}^{\prime}+1$ such that, for all $t \in \mathbb{R}$, we have

$$
\left\|x^{\prime}\right\| \leq M_{2}
$$

In fact, if $x^{\prime}(t)$ is not bounded, then there exists a positive constant $M_{2}^{\prime \prime}$ such that $\left\|x^{\prime}\right\|>M_{2}^{\prime \prime}$ for some $x^{\prime}(t) \in \mathbb{R}$, and therefore we have $\left\|\varphi_{p}\left(x^{\prime}\right)\right\|=\left\|x^{\prime}\right\|^{p-1} \geq\left(M_{2}^{\prime \prime}\right)^{p-1}$, a contradiction, and so Eq. (2.13) holds.

From Eq. (2.3) and Eq. (2.13) we know that there is a point $t_{1} \in[0, T]$ such that $x\left(t_{1}-\right.$ $\left.\sigma\left(t_{1}\right)\right) \geq \gamma_{1}$. Let $\eta_{1}=t_{1}$, where $\eta_{1}$ is as in Eq. (2.3). Then we have

$$
x\left(\eta_{1}-\sigma\left(\eta_{1}\right)\right) \geq \gamma_{1}
$$

where $\gamma_{1}<M_{1}$ is a positive constant independent of $\lambda \in(0,1]$. Meanwhile, we show that for any $t \in[0, T]$, there exits a constant $\gamma_{1}^{\prime} \in\left(0, \gamma_{1}\right)$ such that each positive $T$-periodic solution of Eq. (1.3) satisfies

$$
x(t-\sigma(t))>\gamma_{1}^{\prime} .
$$

On the other hand, we consider the interval $\left[\eta_{1}, t\right] \subset[0, T]$ and $x\left(\eta_{1}-\sigma\left(\eta_{1}\right)\right)>D_{2}$. Multiplying both sides of Eq. (2.2) by $x^{\prime}(t-\sigma(t))\left(1-\sigma^{\prime}(t)\right)$ and integrating on $\left[\eta_{1}, t\right]$, we get

$$
\begin{aligned}
& \int_{\eta_{1}}^{t}\left(\varphi_{p}\left(x^{\prime}(s)\right)\right)^{\prime} x^{\prime}(s-\sigma(s))\left(1-\sigma^{\prime}(s)\right) d s \\
& \quad+\lambda \int_{\eta_{1}}^{t} f\left(s, x^{\prime}(s)\right) x^{\prime}(s-\sigma(s))\left(1-\sigma^{\prime}(s)\right) d s
\end{aligned}
$$




$$
\begin{aligned}
& +\lambda \int_{\eta_{1}}^{t} g_{0}(x(s-\sigma(s))) x^{\prime}(s-\sigma(s))\left(1-\sigma^{\prime}(s)\right) d s \\
& +\lambda \int_{\eta_{1}}^{t} g_{1}(s, x(s-\sigma(s))) x^{\prime}(s-\sigma(s))\left(1-\sigma^{\prime}(s)\right) d s \\
& =\lambda \int_{\eta_{1}}^{t} e(s) x^{\prime}(s-\sigma(s))\left(1-\sigma^{\prime}(s)\right) d s .
\end{aligned}
$$

Furthermore, we have

$$
\begin{aligned}
\left|\lambda \int_{x\left(\eta_{1}-\sigma\left(\eta_{1}\right)\right)}^{x(t-\sigma(t))} g_{0}(v) d v\right| \\
=\lambda\left|\int_{\eta_{1}}^{t} g_{0}(x(s-\sigma(s))) x^{\prime}(s-\sigma(s))\left(1-\sigma^{\prime}(s)\right) d s\right| \\
\leq\left|\int_{\eta_{1}}^{t}\left(\varphi_{p}\left(x^{\prime}(s)\right)\right)^{\prime} x^{\prime}(s-\sigma(s))\left(1-\sigma^{\prime}(s)\right) d s\right| \\
\quad+\lambda\left|\int_{\eta_{1}}^{t} f\left(s, x^{\prime}(s)\right) x^{\prime}(s-\sigma(s))\left(1-\sigma^{\prime}(s)\right) d s\right| \\
\quad+\lambda\left|\int_{\eta_{1}}^{t} g_{1}(s, x(s-\sigma(s))) x^{\prime}(s-\sigma(s))\left(1-\sigma^{\prime}(s)\right) d s\right| \\
\quad+\lambda\left|\int_{\eta_{1}}^{t} e(s) x^{\prime}(s-\sigma(s))\left(1-\sigma^{\prime}(s)\right) d s\right| .
\end{aligned}
$$

By Eq.(2.2) and condition $\left(\mathrm{H}_{1}\right)$ we obtain

$$
\begin{aligned}
& \left|\int_{\eta_{1}}^{t}\left(\varphi_{p}\left(x^{\prime}(s)\right)\right)^{\prime} x^{\prime}(s-\sigma(s))\left(1-\sigma^{\prime}(s)\right) d s\right| \\
& \quad \leq \int_{\eta_{1}}^{t}\left|\left(\varphi_{p}\left(x^{\prime}(s)\right)\right)^{\prime}\right|\left|x^{\prime}(s-\sigma(s))\right|\left|\left(1-\sigma^{\prime}(s)\right)\right| d s \\
& \quad \leq\left(1+\sigma_{0}^{1}\right)\left\|x^{\prime}\right\| \lambda \int_{0}^{T}\left|-f\left(s, x^{\prime}(s)\right)-g(s, x(s, s-\sigma(s)))+e(s)\right| d s \\
& \quad \leq \lambda\left(1+\sigma_{0}^{1}\right) M_{2}\left(2 K T+2 a T\left(M_{1}\right)^{p-1}+2 b T+\|e\| T\right),
\end{aligned}
$$

where $\sigma_{0}^{1}:=\max _{t \in[0, T]}\left(-\sigma^{\prime}(t)\right)$. Meanwhile, we have

$$
\begin{aligned}
& \lambda\left|\int_{\eta_{1}}^{t} f\left(s, x^{\prime}(s)\right) x^{\prime}(s-\sigma(s))\left(1-\sigma^{\prime}(s)\right) d s\right| \leq \lambda\left(1+\sigma_{0}^{1}\right) M_{2} K T \\
& \lambda\left|\int_{\eta_{1}}^{t} g_{1}(s, x(s-\sigma(s))) x^{\prime}(s-\sigma(s))\left(1-\sigma^{\prime}(s)\right) d s\right| \leq \lambda\left(1+\sigma_{0}^{1}\right) M_{2}\left\|g_{1 M_{1}}\right\| T
\end{aligned}
$$

where $\left\|g_{1 M_{1}}\right\|:=\max _{0<x<M_{1}}\left|g_{1}(t, x(t-\sigma(t)))\right|$, and

$$
\lambda\left|\int_{\eta_{1}}^{t} e(s) x^{\prime}(s-\sigma(s))\left(1-\sigma^{\prime}(s)\right) d s\right| \leq \lambda\left(1+\sigma_{0}^{1}\right) M_{2}\|e\| T .
$$


From these inequalities and Eq. (2.14) we derive

$$
\begin{aligned}
\left|\int_{x\left(\eta_{1}-\sigma\left(\eta_{1}\right)\right)}^{x(t-\sigma(t))} g_{0}(v) d v\right| & \leq\left(1+\sigma_{0}^{1}\right) M_{2}\left(3 K T+2 a T\left(M_{1}\right)^{p-1}+2 b T+2\|e\| T+\left\|g_{1 M_{1}}\right\| T\right) \\
& :=M_{3} .
\end{aligned}
$$

In view of the attractive condition (1.4) and $x\left(\eta_{1}-\sigma\left(\eta_{1}\right)\right) \geq \gamma_{1}$, there exists $\gamma_{1}^{\prime} \in\left(0, \gamma_{1}\right)$ such that $\int_{\gamma_{1}^{\prime}}^{\gamma_{1}} g_{0}(v) d v>M_{3}$. Thus, if there is a point $\eta_{1}^{*} \in\left[\eta_{1}, t\right]$ such that $x\left(\eta_{1}^{*}-\sigma\left(\eta_{1}^{*}\right)\right) \leq \gamma_{1}^{\prime}$, then

$$
\left|\int_{x\left(\eta_{1}^{*}-\sigma\left(\eta_{1}^{*}\right)\right)}^{x\left(\eta_{1}-\sigma\left(\eta_{1}\right)\right)} g_{0}(v) d v\right| \geq \int_{\gamma_{1}^{\prime}}^{\gamma_{1}} g_{0}(v) d v>M_{3},
$$

which contradicts Eq. (2.15). Therefore, we obtain that $x(t-\sigma(t))>\gamma_{1}^{\prime}$ for all $t \in[0, T]$.

In the case $t \in\left[0, \eta_{1}\right]$ (i.e., $x(t-\sigma(t)) \in\left[-\sigma(0), \eta_{1}-\sigma\left(\eta_{1}\right)\right]$ ), we can handle similarly.

Define

$$
\Omega=\left\{x \in C_{T}^{1}(\mathbb{R}, \mathbb{R}) \mid E_{1} \leq x(t) \leq E_{2},\left\|x^{\prime}\right\| \leq M_{2}, \forall t \in[0, T]\right\},
$$

where $0<E_{1}<\min \left(D_{2}, \gamma_{1}^{\prime}\right), E_{2}>\max \left(M_{1}, D_{1}\right)$. We know that Eq. (2.2) has no solution on $\partial \Omega$ as $\lambda \in(0,1)$, and when $x(t) \in \partial \Omega \cap \mathbb{R}, x(t)=E_{2}$ or $x(t)=E_{1}$. From Eq. (2.4) we know that $E_{2}>D_{1}$ and $E_{1}<D_{2}$. So, from condition (ii) of Lemma 2.1 we see that

$$
\frac{1}{T} \int_{0}^{T} g\left(t, E_{2}\right) d t<0
$$

and

$$
\frac{1}{T} \int_{0}^{T} g\left(t, E_{1}\right) d t>0
$$

Obviously, we get

$$
\begin{aligned}
\operatorname{deg}\{F, \Omega \cap \mathbb{R}, 0\} & =\operatorname{deg}\left\{\frac{1}{T} \int_{0}^{T} g(t, x) d t, \Omega \cap \mathbb{R}, 0\right\} \\
& =\operatorname{deg}\{x, \Omega \cap \mathbb{R}, 0\} \neq 0,
\end{aligned}
$$

and so condition (iii) of Lemma 2.1 is satisfied. In view of Theorem 2.1, Eq. (1.3) has at least one $T$-periodic solution.

Theorem 2.2 Suppose that condition $\left(\mathrm{H}_{3}\right)$ holds. Assume that the following conditions are satisfied:

$\left(\mathrm{H}_{4}\right) f(t, 0)=0$, and there exist positive constants $m, n$ such that $0 \leq f(t, u) \leq m|u|^{p-1}+n$ for $(t, u) \in \mathbb{R} \times \mathbb{R}$.

$\left(\mathrm{H}_{5}\right)$ There exist constants $D_{3}$ and $D_{4}$ with $0<D_{4}<D_{3}$ such that $g(t, x)<-\|e\|$ for $(t, x) \in$ $\mathbb{R} \times\left(D_{3},+\infty\right)$ and $g(t, x)>\|e\|$ for $(t, x) \in \mathbb{R} \times\left(0, D_{4}\right)$.

Then Eq. (1.3) has at least one solution with period $T$ if $2 m T+2 a T^{p}<1$. 
Proof Consider the homotopic equation

$$
\left(\varphi_{p}\left(x^{\prime}(t)\right)\right)^{\prime}+\lambda f\left(t, x^{\prime}(t)\right)+\lambda g(t, x(t-\sigma(t)))=\lambda e(t) .
$$

We follow the same strategy and notation as in the proof of Theorem 2.1. Let $t^{*}$ and $t_{*}$ be the global maximum point and global minimum point. Since $x(t)$ is $T$-periodic, we get that $x^{\prime}\left(t^{*}\right)=0$ and $x^{\prime}\left(t_{*}\right)=0$. From $\int_{0}^{T}\left(\varphi_{p}\left(x^{\prime}(t)\right)\right)^{\prime} d t=0$ we obtain

$$
\left(\varphi_{p}\left(x^{\prime}\left(t^{*}\right)\right)\right)^{\prime} \leq 0 \text { and }\left(\varphi_{p}\left(x^{\prime}\left(t_{*}\right)\right)\right)^{\prime} \geq 0 .
$$

In fact, if $\left(\varphi_{p}\left(x^{\prime}\left(t_{*}\right)\right)\right)^{\prime} \geq 0$ does not hold, then there exists a constant $\varepsilon>0$ such that $\left(\varphi_{p}\left(x^{\prime}\left(t_{*}\right)\right)\right)^{\prime}<0$ for all $t \in\left(t_{*}-\varepsilon, t_{*}+\varepsilon\right)$. Therefore, $\varphi_{p}\left(x^{\prime}\left(t_{*}\right)\right)$ is strictly decreasing for $\left(t_{*}-\varepsilon, t_{*}+\varepsilon\right)$, and we know that $x^{\prime}(t)$ is strictly decreasing for $\left(t_{*}-\varepsilon, t_{*}+\varepsilon\right)$. This contradicts the definition of $t_{*}$. Thus, we obtain that $\left(\varphi_{p}\left(x^{\prime}\left(t_{*}\right)\right)\right)^{\prime} \geq 0$ is true. From $f(t, 0)=0$ and Eq. (2.16) we have

$$
g\left(t_{*}, x\left(t_{*}-\sigma\left(t_{*}\right)\right)\right)-e\left(t_{*}\right) \leq 0 .
$$

Then, from condition $\left(\mathrm{H}_{5}\right)$ we get that there exists a point $\eta_{2} \in[0, T]$ such that

$$
x\left(\eta_{2}-\sigma\left(\eta_{2}\right)\right) \geq D_{4} .
$$

Similarly, we have

$$
g\left(t^{*}, x\left(t^{*}-\sigma\left(t^{*}\right)\right)\right)-e\left(t^{*}\right) \geq 0 .
$$

Then we get that there exists a point $\xi_{2} \in[0, T]$ such that

$$
x\left(\xi_{2}\right) \leq D_{3} .
$$

Therefore, from $\|x\| \leq x\left(\xi_{2}\right)+T^{\frac{1}{q}}\left(\int_{0}^{T}\left|x^{\prime}(t)\right|^{p} d t\right)^{\frac{1}{p}}$ we get

$$
\|x\| \leq D_{3}+T^{\frac{1}{q}}\left(\int_{0}^{T}\left|x^{\prime}(t)\right|^{p} d t\right)^{\frac{1}{p}} .
$$

From Eq. (2.3) and from conditions $\left(\mathrm{H}_{3}\right)$ and $\left(\mathrm{H}_{4}\right)$ we obtain

$$
\begin{aligned}
& \int_{0}^{T}|g(t, x(t-\sigma(t)))| d t \\
& \quad=\int_{g(t, x(t-\sigma(t)))>0} g^{+}(t, x(t-\sigma(t))) d t-\int_{g(t, x(t-\sigma(t))) \leq 0} g^{-}(t, x(t-\sigma(t))) d t \\
& \quad=-2 \int_{g(t, x(t-\sigma(t))) \leq 0} g^{-}(t, x(t-\sigma(t))) d t+\int_{0}^{T} f\left(t, x^{\prime}(t)\right) d t \\
& \quad \leq 2 \int_{0}^{T}\left(a x^{p-1}+b\right) d t+\int_{0}^{T}\left|f\left(t, x^{\prime}(t)\right)\right| d t \\
& \quad \leq 2 a T\|x\|^{p-1}+2 b T+m \int_{0}^{T}\left|x^{\prime}(t)\right|^{p-1} d t+n T .
\end{aligned}
$$


Then from the Hölder inequality, Eq. (2.6), and Eq. (2.18) we get

$$
\begin{aligned}
\int_{0}^{T}\left|x^{\prime}(t)\right|^{p} d t \leq & 2 a T\|x\|^{p}+2\|x\| m \int_{0}^{T}\left|x^{\prime}(t)\right|^{p-1} d t+\|x\|(2 n T+2 b T+\|e\| T) \\
\leq & 2 a T\|x\|^{p}+2\|x\| m T^{\frac{1}{p}}\left(\int_{0}^{T}\left|x^{\prime}(t)\right|^{p} d t\right)^{\frac{p-1}{p}} \\
& +\|x\|(2 n T+2 b T+\|e\| T) .
\end{aligned}
$$

Substituting Eq. (2.17) into Eq. (2.19), we have

$$
\begin{aligned}
\int_{0}^{T}\left|x^{\prime}(t)\right|^{p} d t \leq & 2 a T\left(D_{3}+T^{\frac{1}{q}}\left(\int_{0}^{T}\left|x^{\prime}(t)\right|^{p} d t\right)^{\frac{1}{p}}\right)^{p} \\
& +\left(D_{3}+T^{\frac{1}{q}}\left(\int_{0}^{T}\left|x^{\prime}(t)\right|^{p} d t\right)^{\frac{1}{p}}\right)(2 n T+2 b T+\|e\| T) \\
& +2\left(D_{3}+T^{\frac{1}{q}}\left(\int_{0}^{T}\left|x^{\prime}(t)\right|^{p} d t\right)^{\frac{1}{p}}\right) m T^{\frac{1}{p}}\left(\int_{0}^{T}\left|x^{\prime}(t)\right|^{p} d t\right)^{\frac{p-1}{p}} \\
\leq & \left(2 m T+2 a T^{p}\right) \int_{0}^{T}\left|x^{\prime}(t)\right|^{p} d t \\
& +\left(2 m D_{3} T^{\frac{1}{p}}+2 a(1+p) D_{3} T^{\frac{p+q-1}{q}}\right)\left(\int_{0}^{T}\left|x^{\prime}(t)\right|^{p} d t\right)^{\frac{p-1}{p}} \\
& +(2 b T+2 n T+\|e\| T) T^{\frac{1}{q}}\left(\int_{0}^{T}\left|x^{\prime}(t)\right|^{p} d t\right)^{\frac{1}{p}} \\
& +(2 b T+2 n T+\|e\| T) D_{3} .
\end{aligned}
$$

Since $2 m T+2 a T^{p}<1$, it is easy to see that there exists a constant $N_{1}^{\prime}>0$ (independent of $\lambda$ ) such that

$$
\int_{0}^{T}\left|x^{\prime}(t)\right|^{p} d t \leq N_{1}^{\prime}
$$

and hence from Eq. (2.20) we have

$$
\|x\| \leq D_{3}+T^{\frac{1}{q}}\left(\int_{0}^{T}\left|x^{\prime}(t)\right|^{p} d t\right)^{\frac{1}{p}} \leq D_{3}+T^{\frac{1}{q}}\left(N_{1}^{\prime}\right)^{\frac{1}{p}}:=N_{1} .
$$

By condition $\left(\mathrm{H}_{4}\right)$ and Eq. (2.12) there exists a constant $N_{2}^{\prime}>0$ such that

$$
\begin{aligned}
\left|\varphi_{p}\left(x^{\prime}(t)\right)\right| & =\left|\int_{t_{0}}^{t}\left(\varphi_{p}\left(x^{\prime}(s)\right)\right)^{\prime} d s\right| \\
& \leq \lambda \int_{0}^{T}\left|f\left(t, x^{\prime}(t)\right)\right| d t+\lambda \int_{0}^{T}|g(t, x(t-\sigma(t)))| d t+\lambda \int_{0}^{T}|e(t)| d t \\
& \leq 2 m T^{\frac{1}{p}}\left(N_{1}^{\prime}\right)^{\frac{p-1}{p}}+2 n T+2 a T N_{1}^{p-1}+2 b T+T\|e\|:=N_{2}^{\prime} .
\end{aligned}
$$


Thus, we obtain that there exists a constant $N_{2}>0$ such that, for all $t \in \mathbb{R}$,

$$
\left\|x^{\prime}\right\| \leq N_{2}
$$

From Eq. (2.3) and Eq. (2.22) we know that there is a point $t_{2} \in[0, T]$ such that $x\left(t_{2}-\right.$ $\left.\sigma\left(t_{2}\right)\right) \geq \gamma_{2}$. Letting $\eta_{2}=t_{2}$, we have

$$
x\left(\eta_{2}-\sigma\left(\eta_{2}\right)\right) \geq \gamma_{2}
$$

where $\gamma_{2}<N_{1}$ is a positive constant independent of $\lambda \in(0,1]$. Meanwhile, we show that, for any $t \in[0, T]$, there exits a constant $\gamma_{2}^{\prime} \in\left(0, \gamma_{2}\right)$ such that each positive $T$-periodic solution of Eq. (1.3) satisfies

$$
x(t-\sigma(t))>\gamma_{2}^{\prime} .
$$

On the other hand, by Eq. (2.2) and condition $\left(\mathrm{H}_{4}\right)$ we obtain

$$
\begin{aligned}
& \left|\int_{\eta_{2}}^{t}\left(\varphi_{p}\left(x^{\prime}(s)\right)\right)^{\prime} x^{\prime}(s-\sigma(s))\left(1-\sigma^{\prime}(s)\right) d s\right| \\
& \quad \leq \int_{\eta_{2}}^{t}\left|\left(\varphi_{p}\left(x^{\prime}(s)\right)\right)^{\prime}\right|\left|x^{\prime}(s-\sigma(s))\right|\left|\left(1-\sigma^{\prime}(s)\right)\right| d s \\
& \quad \leq\left(1+\sigma_{0}^{1}\right)\left\|x^{\prime}\right\| \lambda \int_{0}^{T}\left|-f\left(s, x^{\prime}(s)\right)-g(s, x(s, s-\sigma(s)))+e(s)\right| d s \\
& \quad \leq \lambda\left(1+\sigma_{0}^{1}\right) N_{2}\left(2 m T^{\frac{1}{p}}\left(N_{1}^{\prime}\right)^{\frac{p-1}{p}}+2 n T+2 a T\left(N_{1}\right)^{p-1}+2 b T+\|e\| T\right) .
\end{aligned}
$$

Meanwhile, we have

$$
\begin{aligned}
& \lambda\left|\int_{\eta_{2}}^{t} f\left(s, x^{\prime}(s)\right) x^{\prime}(s-\sigma(s))\left(1-\sigma^{\prime}(s)\right) d s\right| \leq \lambda\left(1+\sigma_{0}^{1}\right) N_{2}\left(m T^{\frac{1}{p}}\left(N_{1}^{\prime}\right)^{\frac{p-1}{p}}+n T\right), \\
& \lambda\left|\int_{\eta_{2}}^{t} g_{1}(s, x(s-\sigma(s))) x^{\prime}(s-\sigma(s))\left(1-\sigma^{\prime}(s)\right) d s\right| \leq \lambda\left(1+\sigma_{0}^{1}\right) N_{2}\left\|g_{1 N_{1}}\right\| T,
\end{aligned}
$$

where $\left\|g_{1 N_{1}}\right\|:=\max _{0<x<N_{1}}\left|g_{1}(t, x(t-\sigma(t)))\right|$, and

$$
\lambda\left|\int_{\eta_{2}}^{t} e(s) x^{\prime}(s-\sigma(s))\left(1-\sigma^{\prime}(s)\right) d s\right| \leq \lambda\left(1+\sigma_{0}^{1}\right) N_{2}\|e\| T .
$$

From those inequalities and Eq. (2.14) we derive

$$
\begin{aligned}
\left|\int_{x\left(\eta_{2}-\sigma\left(\eta_{2}\right)\right)}^{x(t-\sigma(t))} g_{0}(v) d \nu\right| \leq & \left(1+\sigma_{0}^{1}\right) N_{2}\left(3 m T^{\frac{1}{p}}\left(N_{1}^{\prime}\right)^{\frac{p-1}{p}}+3 n T+2 a T\left(N_{1}\right)^{p-1}\right. \\
& \left.+2 b T+2\|e\| T+\left\|g_{1 N_{1}}\right\| T\right):=N_{3} .
\end{aligned}
$$

In view of the attractive condition (1.4) and $x\left(\eta_{2}-\sigma\left(\eta_{2}\right)\right) \geq \gamma_{2}$, there exists $\gamma_{2}^{\prime} \in\left(0, \gamma_{2}\right)$ such that $\int_{\gamma_{2}^{\prime}}^{\gamma_{2}} g_{0}(v) d v>N_{3}$. Thus, if there is a point $\eta_{2}^{*} \in\left[\eta_{2}, t\right]$ such that $x\left(\eta_{2}^{*}-\sigma\left(\eta_{2}^{*}\right)\right) \leq \gamma_{2}^{\prime}$, 
then

$$
\left|\int_{x\left(\eta_{2}^{*}-\sigma\left(\eta_{2}^{*}\right)\right)}^{x\left(\eta_{2}-\sigma\left(\eta_{2}\right)\right)} g_{0}(v) d v\right| \geq \int_{\gamma_{2}^{\prime}}^{\gamma_{2}} g_{0}(v) d v>N_{3}
$$

which contradicts Eq. (2.23). Therefore we obtain that $x(t-\sigma(t))>\gamma_{2}^{\prime}$ for all $t \in$ $[0, T]$.

In the case $t \in\left[0, \eta_{2}\right]$ (i.e., $x(t-\sigma(t)) \in\left[-\sigma(0), \eta_{2}-\sigma\left(\eta_{2}\right)\right]$ ), we can handle similarly.

This proves the claim, and the rest of the proof of the theorem is identical to that of Theorem 2.1.

Example 2.1 Consider the following $p$-Laplacian singular Rayleigh equation with attractive singularity and time-dependent deviating argument:

$$
\begin{aligned}
& \left(\varphi_{p}\left(x^{\prime}(t)\right)\right)^{\prime}+\cos ^{2}(8 t) \sin \left(x^{\prime}(t)\right)-\left(\left(\frac{1}{2} \cos ^{2}(4 t)+\frac{1}{2}\right) x^{5}\left(t-\frac{\cos (8 t)}{11}\right)\right) \\
& +\frac{1}{x^{\mu}\left(t-\frac{\cos (8 t)}{11}\right)}=\sin (8 t),
\end{aligned}
$$

where $p=6$, and $\mu \geq 1$ is a constant.

Comparing Eq. (2.24) to Eq. (1.3), it is easy to see that $f\left(t, x^{\prime}(t)\right)=\cos ^{2}(8 t) \sin \left(x^{\prime}(t)\right)$, so there exists $K=1$ such that $\left|f\left(t, x^{\prime}(t)\right)\right| \leq 1$, and it is obvious that condition $\left(\mathrm{H}_{1}\right)$ holds; $g(t, x(t-\sigma(t)))=-\left(\left(\frac{1}{2} \cos ^{2}(4 t)+\frac{1}{2}\right) x^{5}\left(t-\frac{\cos (8 t)}{11}\right)\right)+\frac{1}{x^{\mu}\left(t-\frac{\cos (8 t)}{11}\right)}, \sigma(t)=\frac{\cos (8 t)}{11}, \sigma^{\prime}(t)=-\frac{8 \sin (8 t)}{11}<$ $1, T=\frac{\pi}{4}$. Since $\frac{1}{p}+\frac{1}{q}=1$, we have $q=\frac{6}{5}$. Consider $g(t, x(t-\sigma(t)))=-\left(\left(\frac{1}{2} \cos ^{2}(4 t)+\frac{1}{2}\right) x^{5}(t-\right.$ $\left.\left.\frac{\cos (8 t)}{11}\right)\right)+\frac{1}{x^{\mu}\left(t-\frac{\cos (8 t)}{11}\right)}$. Then we have $\int_{0}^{1} \frac{1}{x^{\mu}} d x=+\infty$ and $-g(t, x(t-\sigma(t))) \leq x^{5}\left(t-\frac{\cos (8 t)}{11}\right)+1$, where $a=b=1$. So condition $\left(\mathrm{H}_{3}\right)$ is satisfied. Next, we consider the condition

$$
2 a T^{p}=2 \times 1 \times\left(\frac{\pi}{4}\right)^{6} \approx 0.4694
$$

Therefore, by Theorem 2.1 we get that Eq. (2.24) has at least one positive $\frac{\pi}{4}$-periodic solution.

Example 2.2 Consider the following $p$-Laplacian singular Rayleigh equation with attractive singularity and time-dependent deviating argument:

$$
\begin{aligned}
& \left(\varphi_{p}\left(x^{\prime}(t)\right)\right)^{\prime}+\frac{1}{7 \pi}(\sin (12 t)+1)\left(x^{\prime}(t)\right)^{7}-\left(\left(\frac{1}{5} \sin ^{2}(6 t)+\frac{1}{5}\right) x^{7}\left(t-\frac{\sin (12 t)}{18}\right)\right) \\
& +\frac{1}{x^{\mu}\left(t-\frac{\sin (12 t)}{18}\right)}=\cos (12 t),
\end{aligned}
$$

where $p=8$, and $\mu \geq 1$ is a constant.

Comparing Eq. (2.25) to Eq. (1.3), it is easy to see that $f(t, u)=\frac{1}{7 \pi}(\sin (12 t)+1) u^{7}$, so we can choose $m=\frac{2}{7 \pi}$ and $n=1$, so that condition $\left(\mathrm{H}_{4}\right)$ holds; $g(t, x(t-\sigma(t)))=-\left(\left(\frac{1}{5} \sin ^{2}(6 t)+\right.\right.$ $\left.\left.\frac{1}{5}\right) x^{7}\left(t-\frac{\sin (12 t)}{18}\right)\right)+\frac{1}{x^{\mu}\left(t-\frac{\sin (12 t)}{18}\right)}, \sigma(t)=\frac{\sin (12 t)}{18}, \sigma^{\prime}(t)=\frac{2 \cos (12 t)}{3}<1, T=\frac{\pi}{6}$. Since $\frac{1}{p}+\frac{1}{q}=1$, we have $q=\frac{8}{7} ;-g(t, x(t-\sigma(t))) \leq \frac{2}{5} x^{7}\left(t-\frac{\sin (12 t)}{18}\right)+1$, where $a=\frac{2}{5}$ and $b=1$. So, condition $\left(\mathrm{H}_{3}\right)$ 
is satisfied. Next, we consider the condition

$$
2 a T^{p}+2 m T=2 \times \frac{2}{5} \times\left(\frac{\pi}{6}\right)^{8}+2 \times \frac{2}{7 \pi} \times \frac{\pi}{6}=\frac{4}{5} \times\left(\frac{\pi}{6}\right)^{8}+\frac{2}{21} \approx 0.1 .
$$

Therefore, by Theorem 2.2 we see that Eq.(2.25) has at least one positive $\frac{\pi}{6}$-periodic solution.

\section{Conclusions}

In Summary, by Theorems 2.1 and 2.2 we have certified that Eq. (1.3) has at least one $T$-periodic solution. Comparing Theorem 2.1 to Theorem 2.2, the condition $|f(t, u)| \leq$ $a|u|^{p-1}+b$ in Theorem 2.2 is weaker than the condition $|f(t, u)| \leq K$ in Theorem 2.1. Moreover, in view of the mathematical points, the results satisfying conditions of attractive singularity and time-dependent deviating argument are valuable to understand the periodic solutions for Rayleigh equations.

\section{Acknowledgements}

ZBC, ZHB, and SWY would like to thank the referee for invaluable comments and insightful suggestions. Research is supported by National Natural Science Foundation of China (No. 11501170), China Postdoctoral Science Foundation funded project (No. 2016M590886), Fundamental Research Funds for the Universities of Henan Province (NSFRF140142), Henan Polytechnic University Outstanding Youth Fund (J2015-02), and Henan Polytechnic University Doctor Fund (B2013-055).

\section{Competing interests}

The authors declare that they have no competing interests.

\section{Authors' contributions}

ZBC, ZHB, and SWY worked together in the derivation of the mathematical results. All authors read and approved the final manuscript.

\section{Publisher's Note}

Springer Nature remains neutral with regard to jurisdictional claims in published maps and institutional affiliations.

Received: 15 November 2017 Accepted: 31 January 2018 Published online: 09 February 2018

\section{References}

1. Zhang, M.: Periodic solutions of Liénard equation singular forces of repulsive type. J. Math. Anal. Appl. 203, 254-269 (1996)

2. Wang, Z: Periodic solutions of Liénard equation with a singularity and a deviating argument. Nonlinear Anal., Real World Appl. 16(1), 227-234 (2014)

3. Lu, S.: A new result on the existence of periodic solutions for Liénard equation with a singularity of repulsive type. J. Inequal. Appl. 2017, 37 (2017)

4. Cheng, Z., Ren, J.: Periodic and subharmonic solutions for Duffing equation with a singularity. Discrete Contin. Dyn. Syst. 32, 1557-1574 (2012)

5. Chu, J., Torres, P.J., Zhang, M.: Periodic solutions of second order non-autonomous singular dynamical systems. J. Differ. Equ. 239, 196-212 (2007)

6. Fonda, A., Manásevich, R., Zanolin, F.: Subharmonic solutions for some second-order differential equations with singularities. SIAM J. Math. Anal. 24, 1294-1311 (1993)

7. Hakl, R., Torres, P., Zamora, M.: Periodic solution of singular second order differential equations: upper and lower functions. Nonlinear Anal. 74, 7078-7093 (2011)

8. Li, X., Zhang, J.: Periodic solutions for second-order differential equations with a singular nonlinearity. Nonlinear Anal. 69, 3866-3876 (2008)

9. Lu, S., Wang, Y., Guo, Y.: Existence of periodic solutions of a Liénard equation with a singularity of repulsive type. Bound. Value Probl. 2017, 95 (2017)

10. Martins, R.: Existence of periodic solutions for second-order differential equations with singularities and the strong force condition. J. Math. Anal. Appl. 317, 1-13 (2006)

11. Torres, P.: Weak singularities may help periodic solutions to exist. J. Differ. Equ. 232, 277-284 (2007)

12. Wang, Z.H., Ma, T.T.: Existence and multiplicity of periodic solutions of semilinear resonant Duffing equations with singularities. Nonlinearity 25, 279-307 (2012)

13. Xin, Y., Cheng, Z.: Positive periodic solution of $p$-Laplacian Liénard type differential equation with singularity and deviating argument. Adv. Differ. Equ. 2016, 41 (2016)

14. Chen, L., Lu, S.: A new result on the existence of periodic solutions for Rayleigh equations with a singularity of repulsive type. Adv. Differ. Equ. 2017, 106 (2017) 
15. Lu, S., Zhang, T., Chen, L.: Periodic solutions for $p$-Laplacian Rayleigh equations with singularities. Bound. Value Probl. 2016, 96 (2016)

16. Wang, Z., Ma, T.: Periodic solutions of Rayleigh equations with singularities. Bound. Value Probl. 2015, 154 (2015)

17. Manásevich, R., Mawhin, J.: Periodic solutions for nonlinear systems with $p$-Laplacian-like operators. J. Differ. Equ. 145, 367-393 (1998)

Submit your manuscript to a SpringerOpen ${ }^{\circ}$ journal and benefit from:

- Convenient online submission

$\checkmark$ Rigorous peer review

- Open access: articles freely available online

- High visibility within the field

Retaining the copyright to your article

Submit your next manuscript at $\boldsymbol{\triangleright}$ springeropen.com 